\title{
ERRATUM
}

Franka Kahlenberg • Daniel Sanchez •

Ingolf Lachmann • Ludmila Tuckova •

Helena Tlaskalova • Enrique Méndez •

Thomas Mothes

\section{Monoclonal antibody R5 for detection of putatively coeliac-toxic gliadin peptides}

Published online: 21 February 2006

(C) Springer-Verlag 2006

\section{Eur Food Res Technol (2006) 222: 78-82 \\ DOI 10.1007/s00217-005-0100-4}

Due to an unfortunate error, the text on page 80, right-hand column, line 4 was incorrect. The text should read:

"tTG is able to bind biotin-cadaverine to the second amino acid residue in the PQQPFP motif but not to other residues (results not shown)."

The online version of the original article can be found at http//dx.doi.org/10.1007/s00217-005-0100-4

F. Kahlenberg · T. Mothes $(\triangle)$

Institute of Laboratory Medicine, Clinical Chemistry, and

Molecular Disgnostics, University Hospital Leipzig,

Leipzig, Germany

e-mail: mothes@medizin.uni-leipzig.de

Tel.: +49-341-9722251

Fax: +49-341-9722209

D. Sanchez $\cdot$ L. Tuckova $\cdot$ H. Tlaskalova

Department of Immunology, Institute of Microbiology, Czech

Academy of Sciences,

Prague, Czech Republic

I. Lachmann

Roboscreen GmbH Leipzig,

Leipzig, Germany

E. Méndez

Unidad de Gluten, Centre National de Biotecnologia, CSIC,

Campus Universidad Autonoma,

Cantoblanco, Madrid, Spain 